\title{
APROXIMACIÓN AL ESTUDIO DE LA NEUROEDUCACIÓN: EL ENCUENTRO DE LAS CIENCIAS CON LA ESCUELA
}

\author{
Fausto Segovia Baus ${ }^{1}$
}

\section{RESUMEN}

Las neurociencias son las nuevas disciplinas que inciden poderosamente en el conocimiento del funcionamiento del cerebro para enseñar y aprender mejor. En el presente ensayo se pretende conectar la escuela con las neurociencias para incidir en la educación de calidad, y específicamente los aprendizajes.

Palabras clave: neuroeducación, neurodidáctica, ciencias, escuela, investigación.

Una de las debilidades del sistema educativo ecuatoriano es la ausencia de políticas y líneas de investigación, en los ámbitos de la educación, la pedagogía y

\section{ABSTRACT}

Neurosciences are new disciplines deeply involved in our knowledge about brain function and teaching and learning processes. This essay provides a connection between school and neuroscience to improve education, specifically the learning processes.

Key words: neuroscience, neuroeducation, neurodidactic, science, school, research.

la didáctica. Si bien las inversiones en la educación en los últimos años son ostensibles por el financiamiento que ha incrementado la cobertura escolar, la ca-

\footnotetext{
1 Universidad Andina Simón Bolívar, Quito, Ecuador (faustosegovia@cablemodem.com.ec).
} 
lidad constituye una debilidad de origen estructural por los factores complejos que entran en juego.

Los datos oficiales demuestran avances cuantitativos importantes, especialmente en cobertura, pero en los cualitativos -asociados a la calidad- to- davía se reconocen falencias. Informes de la UNESCO pueden ilustrar sobre la situación del país en el concierto internacional, en el ámbito de la educación. El Ecuador se ubicó en la media regional en los resultados TERCE, según la última medición de la UNESCO denominada LLECE (SERCE y TERCE). ${ }^{2}$

\section{Cuadro 1: Distribución de los países según sus resultados y su comparación con la media regional}

\begin{tabular}{|c|c|c|c|c|}
\hline ÁREAS & GRADOS & $\begin{array}{l}\text { POR DEBAJO DE LA } \\
\text { MEDIA REGIONAL }\end{array}$ & $\begin{array}{l}\text { IGUAL QUE LA } \\
\text { MEDIA REGIONAL }\end{array}$ & $\begin{array}{l}\text { POR SOBRE LA } \\
\text { MEDIA REGIONAL }\end{array}$ \\
\hline Lectura & $3^{\circ}$ & $\begin{array}{l}\text { Guatemala, Honduras, } \\
\text { Nicaragua, Panamá, } \\
\text { Paraguay y Rep. } \\
\text { Dominicana }\end{array}$ & $\begin{array}{l}\text { Argentina, Brasil } \\
\text { Colombia y Ecuador }\end{array}$ & $\begin{array}{l}\text { Chile, Costa Rica, } \\
\text { México, Perú, Uruguay, } \\
\text { y el estado mexicanor } \\
\text { de Nuevo León }\end{array}$ \\
\hline Lectura & $6^{\circ}$ & $\begin{array}{l}\text { Ecuador, Guatemala, } \\
\text { Honduras, Nicaragua, } \\
\text { Panamá, Paraguay } \\
\text { y Rep. Dominicana }\end{array}$ & Argentina y Perú & $\begin{array}{c}\text { Brasil, Chile, } \\
\text { Colombia, Costa Rica, } \\
\text { México, Uruguay } \\
\text { y el estado de } \\
\text { Nuevo León (Mex.) }\end{array}$ \\
\hline Matemática & $3^{\circ}$ & $\begin{array}{l}\text { Guatemala, Honduras, } \\
\text { Nicaragua, Panamá, } \\
\text { Paraguay y Rep. } \\
\text { Dominicana }\end{array}$ & Colombia y Ecuador & $\begin{array}{c}\text { Argentina, Brasil, } \\
\text { Chile, Costa Rica, } \\
\text { México, Perú, Uruguay } \\
\text { y el estado de Nuevo } \\
\text { León (Mex.) }\end{array}$ \\
\hline Matemática & $6^{\circ}$ & $\begin{array}{l}\text { Guatemala, Honduras, } \\
\text { Nicaragua, Panamá, } \\
\text { Paraguay y Rep. } \\
\text { Dominicana }\end{array}$ & $\begin{array}{l}\text { Brasil, Colombia } \\
\text { y Ecuador }\end{array}$ & $\begin{array}{c}\text { Argentina, Chile, } \\
\text { Costa Rica, México, } \\
\text { Perú, Uruguay y el estado } \\
\text { de Nuevo León (Mex.) }\end{array}$ \\
\hline $\begin{array}{l}\text { Ciencias } \\
\text { naturales }\end{array}$ & $6^{\circ}$ & $\begin{array}{l}\text { Guatemala, Honduras, } \\
\text { Nicaragua, Panamá, } \\
\text { Paraguay y Rep. } \\
\text { Dominicana }\end{array}$ & $\begin{array}{l}\text { Argentina, Brasil, } \\
\text { Ecuador y Perú }\end{array}$ & $\begin{array}{l}\text { Chile, Colombia, } \\
\text { Costa Rica, México, } \\
\text { Uruguay y el estado } \\
\text { de Nuevo León (Mex.) }\end{array}$ \\
\hline Escritura & $3^{\circ}$ & $\begin{array}{l}\text { Guatemala, Honduras, } \\
\text { Nicaragua, Paraguay } \\
\text { y Rep. Dominicana }\end{array}$ & $\begin{array}{l}\text { Brasil, Colombia, } \\
\text { Ecuador, Panamá } \\
\text { y est. Nuevo León }\end{array}$ & $\begin{array}{l}\text { Argentina, Chile, } \\
\text { Costa Rica, México, } \\
\text { Perú y Uruguay }\end{array}$ \\
\hline Escritura & $6^{\circ}$ & $\begin{array}{l}\text { Colombia, Ecuador, } \\
\text { Honduras, Paraguay } \\
\text { y Rep. Dominicana }\end{array}$ & $\begin{array}{l}\text { Brasil, Nicaragua, } \\
\text { Panamá, Perú y } \\
\text { Uruguay }\end{array}$ & $\begin{array}{l}\text { Argentina, Chile, } \\
\text { Costa Rica, Guatemala, } \\
\text { México y Nuevo León }\end{array}$ \\
\hline
\end{tabular}

Fuente: http://www.unesco.org/new/fileadmin/MULTIMEDIA/FIELD/pdf/TERCE-distribucion-paises-por-resultados.pdf 
Según el Instituto Nacional de Evaluación (INEVAL), si se comparan los resultados entre las pruebas TERCE ejecutadas en 2013 por el LLECE de la UNESCO, y también la prueba precedente -SERCE 2006- "el Ecuador se encuentra entre los países con más avances en los aprendizajes". Y subraya que "es importante destacar que el incremento de los puntajes en el desempeño educativo de Ecuador va acompañado de una mejor distribución de la riqueza, lo cual se evidencia con el índice Gini que pasó de 0,53 en el 2006 a 0,46 en el 2011; y de un incremento en el gasto público destinado a la educación que pasó de 2,6 \% en el 2006 a $5 \%$ en el 2013. En el caso del Índice de Desarrollo Humano (IDH) de 0,687 (2005) a 0,711 (2013). Lo que significa que se ha alcanzado un desarrollo humano medio alto".3
Un punto importante digno de mencionarse es la ejecución de las ocho políticas educativas de Estado contenidas en el Plan Decenal de Educación 2006-2015, que fueron aprobadas en referendo por el pueblo ecuatoriano, que constituyeron una guía significativa para el desarrollo de los planes, programas y proyectos en marcha.

La información anterior establece -en resumen- que el Ecuador en los últimos diez años ha realizado esfuerzos importantes en el sector educativo gracias a algunos factores: voluntad política, altas inversiones, reformas legales y la creación de cuatro nuevas universidades: UNAE, Universidad Nacional de Educación; IKIAM, Universidad Amazónica; UNIARTES, Universidad de las Artes; y YACHAY, Universidad de Investigación y Tecnología Experimental.

\section{PARADOJAS}

Estos emprendimientos se inscribieron en el Plan de Desarrollo del "Buen Vivir", que ubicó a la reforma educativa en uno de los cinco ejes de la denominada Revolución Ciudadana, y donde los actores directos e indirectos del sistema educativo tuvieron escasa participación.
Prueba de lo mencionado es la no convocatoria, por ejemplo, al Consejo Nacional de Educación, según el Art. 23 de la Ley Orgánica de Educación Intercultural, cuya disposición es mandataria u obligatoria y no facultativa. ${ }^{4}$

2 LLECE es el Laboratorio Latinoamericano de Evaluación de la Calidad de la Evaluación, coordinado por la Oficina Regional de la UNESCO. EI TERCE se aplicó en 15 países de América Latina: Argentina, Brasil, Chile, Colombia, Costa Rica, Ecuador, Guatemala, Honduras, México, Nicaragua, Panamá, Paraguay, Perú, República Dominicana y Uruguay, y en el estado mexicano de Nuevo León.

${ }^{3}$ SÁNCHEZ, Harvey. Director Ejecutivo de INEVAL. En documento electrónico TERCE -INEVAL-UNESCO. Disponible en: http://www.unesco.org/new/fileadmin/MULTIMEDIA/FIELD/Santiago/pdf/TERCE-distribucion-paises-por-resultados.pdf

${ }^{4}$ REGISTRO OFICIAL DE LA REPÚBLICA DEL ECUADOR. n. 417, de 31 de marzo de 2011. Disponible en: http://planipolis.iiep.unesco.org/upload/Ecuador/Ecuador_Ley_organica_educacion_intercultural.pdf 
Pero lo paradójico del proceso estriba no solo en la poca o escasa participación de los actores sociales, especialmente de los profesores; por ejemplo, en validaciones técnicas y consensos cardinales, sino en la ausencia de las universidades y sus centros de investigación, en el diseño y ejecución de las políticas públicas en educación y en la fundamentación científica de las reformas.
En esa línea de pensamiento, un punto en discusión -todavía intocado en el Ecuador- es la necesaria conexión de la escuela con las ciencias, como núcleo central de la educación inicial y continua de los docentes y específicamente de los sistemas neurolingüísticos asociados a la lectura, que es el tema que motiva este artículo.

\section{NECESIDAD DE INTERCONEXIONES}

En el Ecuador existen pocos estudios sobre las neurociencias y sus relaciones con las escuelas y los procesos de aprendizaje de los estudiantes. Las escuelas -salvo excepciones- actúan sobre sistemas formales, anclados a modelos tradicionales, donde la gestión del currículo -lo administrativo- supera a las teorías pedagógicas, así como a los fundamentos curriculares, que deberían formar parte de la actualización de los docentes, y a los estándares de aprendizaje de los estudiantes, sobre la base de sistemas de evaluación y calidad sustentados en parámetros científicos.

Tracey Tokuhama-Espinosa ${ }^{5}$ ha identificado las interconexiones entre la psicología, el cerebro y la educación como ejes de sus investigaciones y publicaciones, que tienden a fortalecer la calidad de la educación desde perspec- tivas científicas. Sus trabajos han sido reconocidos por la comunidad educativa internacional.

Y si nos referimos a la escuela, los vacíos son evidentes tanto en lo conceptual y pedagógico. La escuela del Ecuador responde a sistemas tradicionales de enseñanza y aprendizaje, y su estructura reproduce un sistema social de carácter piramidal y paternalista, cuya cultura organizacional privilegia la memorización de cantidades de "materia", en nombre de un currículo inflexible, y donde los modelos de evaluación fomentan la calificación y generan incertidumbres. A lo anterior se une la falta de programas de investigación asociados a la educación, la pedagogía y la didáctica para la toma de decisiones estratégicas y la construcción de políticas públicas. Y si existen, la mayoría de las investigacio-

${ }^{5}$ Dra. Tracey Tokuhama-Espinosa, Ph.D. Director of IDEA (Instituto de Enseñanza y Aprendizaje or Teaching and Learning Institute), and Professor of Education and Neuropsychology at the of the University of San Francisco in Quito, Ecuador. 
nes (las tesis de grado y posgrado son ejemplos) quedan en los archivos de las bibliotecas de las universidades. La Ley Orgánica de Educación Intercultural menciona a la escuela, pero con énfasis en la gestión, mas no en lo pedagógico y cualitativo.
Las neurociencias y la programación neurolingüística, en este contexto, no pasan de ser temas que se estudian en cursos especiales, sin mayor incidencia en las políticas públicas.

\section{LA NEUROEDUCACIÓN}

La pedagogía, la psicología y las neurociencias -entre las que se destacan la neuroeducación y la neurodidáctica- son nuevos enfoques y corrientes de investigación que, en su conjunto, tienen como objeto de estudio la formación integral de los seres humanos, de manera especial en conexión con los aprendizajes, el funcionamiento del cerebro y los comportamientos.

En la actualidad la neuroeducación es considerada una disciplina -o mejor, una transdisciplina- que integra varias ciencias de la educación, cuyo núcleo central es el estudio del cerebro como constructo interdisciplurocognitivo de los seres humanos. Su vigencia es reciente. En 1971, Arthur Benton publicó el libro Introducción a la neuropsicología. De ahí surgieron otras investigaciones sobre neuropsicología infantil y sus derivaciones: la neuropsicología del aprendizaje, la neuropsicología escolar, la neuropedagogía y la neurodidáctica. En 1988, Gerhard Preiss, investigador de la Universidad de Friburgo, intro- dujo la neurodidáctica como ciencia autónoma encargada del estudio de las relaciones entre el cerebro y la pedago gía ${ }^{6}$.

Los recursos de alta tecnología han permitido el desarrollo extraordinario de la neuroeducación en los últimos años, a saber: las imágenes cerebrales, las pruebas genéticas, las simulaciones computacionales, entre otras, cuya utilización va más allá de los trastornos del aprendizaje, discapacidades, dislexia, autismo y defectos de atención. La neuroeducación intenta articular las ciencias del cerebro -conocidas como neurociencias- con las ciencias de la educación.

Tracey Tokuhama-Espinosa concibe así la neuroeducación, según el libro Ciencias de la educación, mentey cerebro: una guía completa a la nueva enseñanza basada en el cerebro (W.W. Norton), basado en más de 4.500 estudios y contribuciones de los líderes del mundo, en la nueva educación centrada en el estudio del cerebro:

\footnotetext{
${ }^{6}$ Neuroeducación. Disponible en: https://es.wikipedia.org/wiki/Neuroeducaci\%C3\%B3n
} 


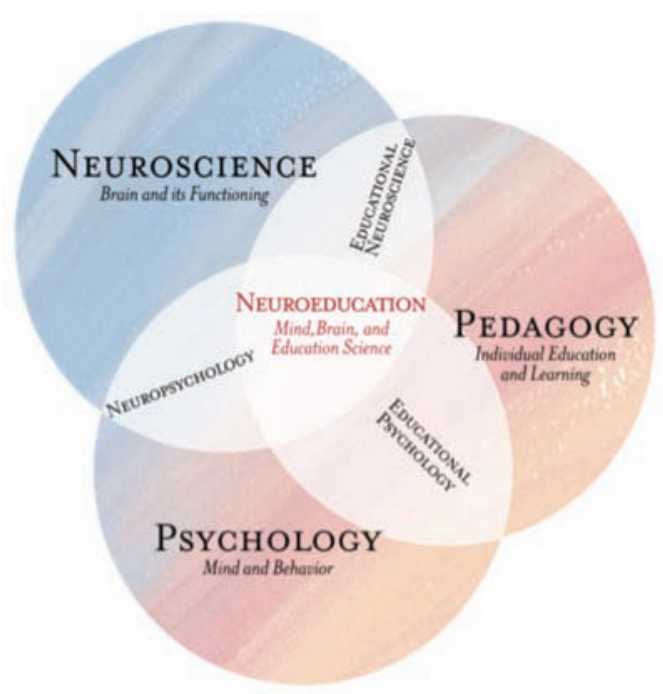

Fuente: MBE Science as a Multidisciplinary Field. Interpretación en línea transdisciplinaria, por TokuhamaEspinosa Nakagawa, (2008), redibujado por Bramwell, (2010).

La neuroeducación se nutre de las neurociencias, que explican las conexiones y funciones del cerebro; de la psicología o ciencia de los comportamientos humanos asociados a la mente y el pensamiento crítico o cognición; y a la pedagogía, cuyo centro de atención son los aprendizajes.

\section{EL CEREBRO LECTOR}

El ser humano tiene entre 12 y 15 mil millones de células nerviosas o neuronas, aproximadamente. Cada neurona, a su vez, puede construir millones de conexiones, que se forman al usarse el cerebro. Así, cuando son más estimuladas se construyen más conexiones y más capacidades del ser humano para pensar. Por eso la lectura es considerada pensamiento en acción.
El cerebro humano, en efecto, es un órgano maravilloso dotado de una complejidad impresionante. Sus funciones neurocognitivas retratan la historia evolutiva de las personas y de las diferentes especies mediante estudios comparados.

Las neurociencias, en este sentido, recién comienzan a investigarse, y en el 
caso de la neuroeducación su interdisciplinariedad es indispensable, así como una rigurosa confirmación experimental, junto con la'neuroética', que permita evitar falsificaciones o manipulaciones inaceptables.

Uno de los puntos relevantes que se debe considerar, en el ámbito de la neurodidáctica, es la enseñanza y aprendizaje de la lectura. En este contexto, los trabajos de Stanislas Dehaene ${ }^{7}$ son relevantes. En esta investigación, el autor da a conocer las últimas noticias de las neurociencias sobre la lectura, la enseñanza, el aprendizaje y la dislexia.

La arquitectura cerebral es similar en todos los miembros de la familia de los Homo Sapiens, y se diferencia muy poco de la de otros primates. A lo largo y a lo ancho del mundo, las mismas regiones cerebrales se activan para decodificar una palabra escrita. Ya se trata de francés o de chino, el aprendizaje de la lectura recorre un circuito genéticamente condicionado (Dehaene, 2014).

La importancia de la lectura -como sabemos- va mucho más allá de enseñar a leer. El reduccionismo, a veces, ha matado la lectura y por extensión a la calidad de la educación. Los educadores enseñamos a leer -y es un mérito relevante- bajo sistemas que aprendimos en los normales y en las universidades. Pero hoy, en el siglo XXI, el tema de la lectura ha superado los modelos tradicionales que están centrados en la lectura mecánica, orientados a adquirir una serie de automatismos que permiten a los estudiantes a interpretar signos gráficos, mediante una percepción visual y darles una identidad oral. Se insiste más en la lectura comprensiva, que tiende a asociar las letras con los sonidos y las palabras con su significado, y lograr, en última instancia, la lectura crítica.

Existen varios métodos de enseñanza de la lectura. Los más conocidos son el método fónico, el método global y el método constructivista. El primero se fundamenta en el principio alfabético, que implica la asociación entre fonemas y grafemas, y de las vocales a las consonantes. El segundo se centra en las palabras, que deben ser memorizadas inicialmente, como base de la creación de los primeros enunciados, cuyos significados son aprendidos con la ayuda de dibujos, conocimientos previos y más. De esta manera, las palabras antes desconocidas cobran sentido. Por último, el método constructivista, creado por Jean Piaget, sugiere la enseñanza de la lectura a partir de las hipótesis implícitas que el niño desarrolla del aspecto fonológico. Pero el debate entre estos modelos de enseñanza de la lectura no

\footnotetext{
${ }^{7}$ Stanislas Dehaene es uno de los científicos más representativos, que ha estudiado con rigor el tema del "cerebro lector", en el ámbito de las neurociencias.
} 
ha terminado, porque existen evidencias empíricas que muestran la importancia de la conciencia fonológica en el proceso de alfabetización, y que es posible integrar los métodos fónico y global.

Lo anterior sería imposible entender sin considerar la existencia de un cerebro lector, de acuerdo con la terminología de Stanislas Dehaene; es decir, de la arquitectura cerebral que hace posible las relaciones entre las neuronas que explican las neurociencias, y permiten, según Max Weber:"saber pronunciar las palabras escritas; saber identificar las palabras y el significado de cada una de ellas; y, saber extraer y comprender el significado de un texto".

El tema es complejo y fascinante. Para algunos investigadores estamos todavía en la "madrugada" de la palabra es- crita, porque recién comienza a descifrarse en el ADN de la lectura y algunos campos específicos de la neuro educación: el aprendizaje, la memoria, la atención, la dislexia, entre otros asuntos, donde la bioingeniería, la computación y la biología molecular se han unido para cambiar para siempre a la pedagogía y la medicina.

Las ciencias aplicadas a los aprendizajes están, por lo tanto, en "pañales". Pese a ello, en los últimos veinte años, la pedagogía y la psicología, con el apoyo de las neurociencias, han podido generar mapas preliminares del cerebro, en los cuales se identifican los circuitos cerebrales de la lectura, a través de neuro imágenes o imágenes cerebrales, que revelan las áreas que se activan cuando desciframos palabras escritas.

\section{¿LA CIENCIA DE LA LECTURA?}

Stanislas Dehaene -ya mencionado- considera que 'una persona promedio sabe más sobre su auto, que sobre el funcionamiento de su cerebro's.

La nueva ciencia de la lectura es desconocida en varias partes del mundo, y peor aplicada. Las razones son obvias: la mayoría de los modelos de enseñanza y aprendizaje -y en general de las cienciasson lineales, mecanicistas-funcionalistas, que reducen los fenómenos a mecanismos limitados o reduccionistas; por ejemplo, células, moléculas o modelos de conducta de los seres vivientes. Cuando el enfoque dinámico de los sistemas considera al mundo a partir de vínculos e integración.

\footnotetext{
${ }^{8}$ Neuroeducación. Disponible en: https://es.wikipedia.org/wiki/Neuroeducaci\%C3\%B3n

${ }^{9}$ Es una aseveración muy cierta: la mayoría de las personas sabe muy poco sobre su propio cuerpo, mientras domina ciertas herramientas de uso diario. El cerebro, en este contexto, es casi desconocido.
} 
En otras palabras, todo es sistémico y sus estructuras específicas resultan de las relaciones entre las partes o subsistemas. En este contexto, la lectura es un sistema complejo cuyo eje es el cerebro, que es la central de manejo, que actúa también como centro de la memoria.

\section{LA NEUROEDUCACIÓN EN LA ESCUELA}

No se trata de convertir a los médicos neurólogos en neuropedagogos; tampoco a los pedagogos en médicos especialistas en neurología. La idea central es crear espacios académicos interdisciplinares para investigar el cerebro que aprende... matemáticas o física, el cerebro bilingüe o el cerebro que aprende las artes o las ciencias sociales.

Hoy en día las investigaciones se realizan fuera de las escuelas, en los laboratorios; es decir, en ambientes controlados, por ejemplo, los hospitales. No obstante, se pretende a futuro llevar la neuroeducación a las aulas, al identificar los procesos neurocognitivos de ense- ñanza y aprendizaje, mediante sistemas de información y comunicación de alta tecnología, que ayuden a contar, por ejemplo, con imágenes cerebrales de profesores y estudiantes en interacción. Para ello-sugieren los especialistas- es necesario crear comités de neuroética en las escuelas, así como existen comités de bioética en los hospitales.

El camino está abierto. La Escuela de Educación de la Universidad de Harvard ofrece cursos anuales sobre mente, cerebro y educación. En el Ecuador, el Instituto IDEA, de la Universidad de San Francisco de Quito, ejecuta cursos de alta calidad sobre estos temas.

\section{APUNTES PARA UNA INVESTIGACIÓN INTERDISCIPLINAR}

El tema planteado lleva a un propósito central: articular la ciencias -las neurociencias con la escuela-, a través de modelos de investigación aplicada en los que se integren la pedagogía, la psicología y las neurociencias, y dentro de este esquema analizar los problemas recurrentes de la lectura y la escritura, que son, en su conjunto, situaciones estructurales de la educación ecuatoriana.
Recuérdese que, pese a los esfuerzos realizados en inversiones y equipamiento, la calidad de la educación es todavía una asignatura pendiente. Los estudios de neuroeducación asociados a la calidad, y en este contexto, de manera específica, a la lectura y escritura, podrían ayudar a descifrar los problemas de los estudiantes que, en el nivel básico, tienen dificultades de compresión 
lectora. Y también en el proceso de selección y formación inicial y continua de los docentes.

La receta no es la neuroeducación; tampoco la neurodidáctica. Es importante incorporar la investigación -rigurosamente concebida y aplicada-, con la participación activa de las universidades, en la construcción de nuevas teorías y métodos que acompañen los procesos de cambio educativo. Lo hecho hasta aquí es relevante, en cuanto a infraestructura y equipamiento, pero falta dar fundamento teórico-epistemo- lógico-metodológico a los procesos de reforma del currículo de los estudiantes y de la formación de los profesores.

El nuevo Plan Decenal de Educación 2016-2026 debería incorporar la investigación educativa de carácter interdisciplinaria, que integre las neurociencias, la pedagogía y la psicología, en la formación del profesorado y como parte esencial de una nueva política pública con el compromiso de la academia. Y que el encuentro de las ciencias con la escuela no sea una utopía más. 


\section{BIBLIOGRAFÍA}

Dehaene, S. (2014) El cerebro lector. Siglo XXI Editores. Ciencia que labra. Serie Mayor. Buenos Aires, Argentina. pp 9-45.

Tokuhama-Espinosa, T. (2008) http:// education.jhu.edu/PD/newhorizons/Journals/Winter2011/Tokuhama1

Sánchez, Harvey. Director Ejecutivo de INEVAL. http://www.unesco.org/new/fileadmin/MULTIMEDIA/FIELD/Santiago/pdf/TERC E-distribucion-paises-por-resultados.pdf

Registro Oficial del Ecuador. n. ${ }^{\circ} 417$, de 31 de marzo de 2011. http://planipolis.iiep .unesco.org/upload/Ecuador/Ecuador_Ley_ organica_educacion_intercultural.pdf http://www.unesco.org/new/fileadmin/MULTIMEDIA/FIELD/Santiago/pdf/TERC E-distribucion-paises-por-resultados.pdf

http://my.gse.harvard.edu/icb/icb.do? course $=$ gse-ht100

http://www.znl-ulm.de

http://www.tc.umn.edu/ athe0007/B NEsig/ http://w3.cnice.mec.es/oecd/department/cerebro.html

http://my.gse.harvard.edu/icb/icb.do? course $=$ gse $-\mathrm{ht} 100$

https://es.wikipedia.org/wiki/Neuroeducaci\%C3\%B3n 